\title{
"Si una mujer avanza, ningún hombre retrocede": memorias de las mujeres en los movimientos territoriales de la década del 90 en Argentina. Testimonios y archivos en una colección de Memoria Abierta
}

\author{
"If a Woman Goes Forward, No Man Backs Away": Memories of Women in \\ Territorial Movements During the Nineties in Argentina. Testimonies and \\ Archives in a Memoria Abierta Collection \\ "Ma mulher avança, nenhum homen retrocede": Memórias das mulheres nos \\ movimentos territoriais da década de 90 na Argentina. Testemunhos e arquivos \\ de uma coleção de Memória aberta
}

\begin{abstract}
Maisa Bascuas
mbascuas.memoriaabierta@gmail.com

Universidad de Buenos Aires - Memoria Abierta,

Argentina
\end{abstract}

\section{Victoria Daona \\ vdaona.memoriaabierta@gmail.com \\ Instituto de Desarrollo Económico y Social - Memoria \\ Abierta, Argentina}

Nancy Lucero

nlucero.memoriaabierta@gmail.com

Memoria Abierta, Argentina

\section{Alejandra Oberti}

aoberti.memoriaabierta@gmail.com

Universidad de Buenos Aires - Memoria Abierta,

Argentina

Cita sugerida: Bascuas, M., Daona, V., Lucero, N. y Oberti, A. (2021). "Si una mujer avanza, ningún hombre retrocede": memorias de las mujeres en los movimientos territoriales de la década del 90 en Argentina. Testimonios y archivos en una colección de Memoria Abierta. Aletheia, 12(23), e117. https://doi.org/10.24215/18533701e117
Resumen: La II Conferencia Internacional El lugar del archivo de las violencias de Estado: reorganizaciones del poder bajo el neoliberalismo se desarrolló los días 2, 3, 9 y 10 de septiembre de 2021. Fue organizada por el Proyecto Anillos de Investigación "Tecnologías Políticas de la memoria: usos y apropiaciones contemporáneas de artefactos de registro de la violencia política”, de la Universidad Alberto Hurtado, el Museo de la Memoria y los Derechos Humanos y la Universidad Austral de Chile. A su vez, contó con la colaboración de Memoria Abierta (Argentina), el grupo de investigación "Representaciones contemporáneas de perpetración de crímenes de masas" (España) y el Núcleo Milenio Arte, Performatividad y Activismo (Chile). En ese marco y como parte del panel "Activismos y registros feministas", se presentó la ponencia "Si una mujer avanza, ningún hombre retrocede”, desarrollada por el equipo de trabajo del proyecto Insumisas de Memoria Abierta. La presentación que, a continuación compartimos, estuvo a cargo de Maisa Bascuas, Victoria Daona y Nancy Lucero y fue moderada por Alejandra Oberti quien junto a Verónica Torras coordinan el proyecto

Palabras clave: Archivo, Feminismos, Derechos Humanos.

Abstract: The Second International Conference El lugar del archivo de las violencias de Estado: reorganizaciones del poder bajo el neoliberalismo (The Place of State Violence Archives: Reorganizations of Power Under Neoliberalism) took place on September 2, 3, 9 and 10, 2021. It was organized by the Research Project "Tecnologías Políticas de la memoria: usos y apropiaciones contemporáneas de artefactos de registro de la violencia política" (Political Technologies of Memory: 
contemporary uses and reappropriations of artifacts that record political violence), from Universidad Alberto Hurtado (Chile), the Museo de la Memoria y los Derechos Humanos (Memory and Human Rights Museum) in Chile, and the Universidad Austral de Chile. Institutions such as Memoria Abierta (Open Memory), from Argentina, the research group "Representaciones contemporáneas de perpetración de crímenes de masas" (Contemporary Representations of the Perpetration of Mass Crimes), from Spain, and the "Núcleo Milenio Arte, Performatividad y Activismo" (Millennium Art, Performativity, and Activism Group), from Chile. In the context of its panel "Activismos y registros feministas" (Activisms and Feminist Records), the presentation "Si una mujer avanza, ningún hombre retrocede" (If a woman goes forward, no man backs away) was delivered by the Memoria Abierta Insumisas (Unsubmissive) project team. Thepresenters were Maisa Bascuas, Victoria Daona and Nancy Lucero, with moderation by Alejandra Oberti, project coordinator in conjunction with Veronica Torras.

Keywords: Archive, Feminisms, Human rights.

Resumo: A II Conferência Internacional O lugar do arquivo das violências de Estado: reorganizaçôes do poder sob o neoliberalismo teve lugar nos dias 2, 3, 9 e 10 de setembro de 2021. Foi desenvolvida pelo ProjectoAnillos de Investigación "Tecnologias Políticas da memória: usos e apropriações contemporâneas de dispositivos de registro da violência política”, da Universidad Alberto Hurtado, pelo Museo de la Memoria y Derechos Humanos e pela Universidad Austral do Chile. Ao mesmo tempo, contou com a colaboração de Memoria Abierta (Argentina), do grupo de investigação "Representações contemporâneas de perpetração de crimes de masas" (Espanha) e do Núcleo Milenio Arte, Performatividad y Activismo (Chile). Nesse marco e como parte do painel "Ativismos e registros feministas" foi apresentada a palestra "Se uma mulher avança, nenhum homem retrocede", desenvolvida pela equipe de trabalho do projeto Insumisas de Memoria Abierta. A apresentação que compartilhamos a seguir esteve a cargo de Maisa Bascuas, Victoria Daona e Nancy Lucero, e foi moderada por Alejandra Oberti quem coordena o projeto junto com Verónica Torras.

Palavras-chave: Arquivo, Feminismos, Direitos Humanos.

Alejandra Oberti: A continuación, escucharemos el trabajo "Si una mujer avanza, ningún hombre retrocede: memorias de las mujeres en los movimientos territoriales de la década del 90 en Argentina”. La presentación estará a cargo de dos integrantes del equipo que están desarrollando el proyecto Insumisas de Memoria Abierta. Maisa Bascuas, es argentina, estudió Ciencias Políticas en la Universidad de Buenos Aires y es miembro del grupo de estudios sobre Feminismos en América Latina. Nancy Lucero, también es argentina, fotógrafa y archivista audiovisual, ambas son parte del archivo oral de Memoria Abierta. 
Nancy Lucero: Muchas gracias por la presentación y por el espacio para compartir el trabajo. Como comentó Alejandra, vamos a hablar de los vínculos entre el movimiento de derechos humanos y el movimiento de mujeres, de sus encuentros, sus diálogos, sus estrategias y alianzas que no siempre han sido tan visibles.

El esfuerzo de militantes e investigadoras por reinscribir a las mujeres en el campo de la historia -por ejemplo, la historia de las mujeres, los estudios de género, la crítica feminista de los saberes- se ha ocupado de la tarea indispensable de analizar el lugar subordinado de las mujeres en la producción y en los espacios de poder y, a la vez, de recuperar los invisibilizados aportes femeninos a la historia colectiva. En esta línea de trabajo, se inscribe Memoria Abierta con su colección Insumisas. Intentamos reconstruir, por un lado, la genealogía de la articulación entre el movimiento de mujeres y el movimiento de derechos humanos en las acciones, los grupos, las militancias cruzadas y las discusiones; y por otro, el modo en que el movimiento de derechos humanos dejó su huella en las formas del activismo feminista. Una impronta que se puede rastrear tanto en los modos de intervención y el lenguaje, como en la producción de campo de derechos humanos ampliado.

En este cruce, nos encontramos con una cuestión fundamental para el proyecto: la cuestión del testimonio que, desde Memoria Abierta, lo comprendemos como la narración y el relato del pasado que se recupera desde claves interpretativas del presente. En ese sentido, el testimonio es una narración que vuelve sobre problemas del pasado desde las posibilidades del presente para hacer audible nuevas claves interpretativas y releer esos procesos desde allí. Es ante todo una narración en primera persona que da cuenta de una subjetividad marcada por situaciones límite. Al volver sobre problemas del pasado a la luz de conceptos, dilemas y formas de abordaje que son propias del presente, el testimonio constituye una fuente de información y de contenido que se transforma en archivo.

En el archivo oral de Memoria Abierta recogemos testimonios desde el año 2001. Esos primeros relatos estuvieron inicialmente relacionados al terrorismo de Estado durante la última dictadura y a sus consecuencias en la vida social y política, así como en las vidas particulares de quienes fueron víctimas, directa o indirectamente, de ese accionar. Se trata de testimonios que dan cuenta de las diferentes maneras en que se desarrollaron estrategias de resistencia a nivel del movimiento de derechos humanos, desde los organismos hasta las personas individuales afectadas de alguna manera. En este camino, los testimonios orales de la colección Insumisas fueron recogidos hace pocos años y se han orientado a recuperar, entre otras cosas, la transmisión de prácticas y estrategias llevadas adelante por el movimiento de derechos humanos en el cruce con el desarrollo del movimiento de mujeres desde sus distintos enfoques y desde sus diferentes actores: militantes de movimientos sociales y territoriales, integrantes del campo académico, grupos feministas y promotoras de derechos. En otras palabras, se trata de testimonios en donde también se inscribe el nombre de otras mujeres que han tenido trayectorias o historias similares respecto al padecimiento de diversas formas de la violencia y de sus resistencias. Y los caminos que esas mujeres fueron recorriendo en la lucha por lograr los cambios necesarios en pos de una sociedad más igualitaria, han quedado registrados en estos relatos.

Asimismo, en varias ocasiones estos testimonios fueron acompañados por archivos personales que daban cuenta del recorrido transitado en los reclamos por los derechos incumplidos o negados por la sociedad patriarcal. Se trata de documentos en papel, publicaciones, fotografías, grabaciones en casetes de audio, que han fortalecido el contenido de los relatos orales. Estos archivos fueron compartidos al momento de la entrevista y por iniciativa de las mismas entrevistadas.

Si hablamos de las estrategias y de los modos de organización de las integrantes del movimiento de derechos humanos, las Madres de Plaza de Mayo han sido y siguen siendo referentes muy potentes para la lucha de las mujeres en todo sentido. $Y$ en ese punto, nos interesa rescatar una lectura feminista de las Madres, que habilitaron la producción de tramas, encuentros y de renovadas reflexiones para el movimiento de mujeres.

La vuelta de la democracia en 1983 marca la irrupción en la escena pública de un nuevo movimiento en construcción, el movimiento de mujeres y feminista. Con un lenguaje anclado en la demanda de derechos se constituye como un espacio que se va tejiendo de distintas alianzas entre grupos feministas y de mujeres 
con partidos políticos, sindicales y, especialmente, de derechos humanos. Por ejemplo, la construcción de la Multisectorial de la Mujer y la organización de la jornada pública en la Plaza de los Dos Congresos el 8 de marzo de 1984 - el primer 8 de marzo conmemorado públicamente en Argentina - forma parte de esos esfuerzos por habilitar la voz y la participación de las mujeres en la reconstrucción de la democracia. Desde esos primeros momentos, diversos grupos feministas como ATEM "25 de noviembre", promueve acercamientos y construye espacios de encuentro con las referentes del movimiento de derechos humanos. Estos grupos producen una relectura de la práctica política de resistencia de las Madres de Plaza de Mayo contra la dictadura y el terrorismo de Estado que la conciben como una forma práctica de rehabitar la maternidad desde una perspectiva política y de resistencia. Desde esta clave de lectura feminista, rescatan el protagonismo de las mujeres en la búsqueda de sus hijos/as, de sus nietos/as y familiares que "dejan la cocina", podemos decir, "para romper el silencio". En ese proceso, se encuentran con otras y otros en la búsqueda de verdad y justicia y se convierten en referencias ineludibles en la lucha contra la dictadura. Las Madres no se han definido en términos feministas pero, a su modo, han tensionado ciertos elementos nodales del patriarcado.

También, en este contexto de mediados de los años ochenta, se fueron produciendo otros procesos sociales y políticos que tuvieron a las mujeres como actores privilegiados en la construcción de nuevas formas de resistencia y demandas de derechos humanos. Nos referimos a los procesos de recuperación de tierras, ocupación de territorio, de resistencias a los desalojos, a la violencia y a la estigmatización. Sobre estas tramas y cruces, nos hablará Maisa.

Maisa Bascuas: Retomando lo que decía Nancy, este trabajo de archivo y de producción de testimonios que, ante todo, pone en evidencia los encuentros entre el movimiento de mujeres y el de derechos humanos, también nos ha permitido identificar un conjunto de referentes de aquellos procesos de organización de mediados de los ochenta. También allí encontramos puntos de conexión entre ambos movimientos.

Desde comienzos de los años ochenta, se extendió por diferentes partidos del conurbano bonaerense el fenómeno de tomas de tierra por parte de sectores populares afectados por la falta de vivienda. Las tomas y la construcción de asentamientos resultaron en procesos organizativos que se valieron de diversas experiencias formativas sindicales, partidarias, eclesiales, internacionales y de los organismos de derechos humanos. El apoyo de las Comunidades Eclesiales de Base y de los organismos de derechos humanos (especialmente la APDH y el SERPAJ) resultaron fundamentales para la organización y defensa de esos espacios. En este proceso, la ocupación de tierras produjo nuevos saberes y formas de organización y lucha porque, además de una forma de solucionar el acceso a la vivienda, éstas resultaron una estrategia comunitaria de mejora de las condiciones de vida para amplios sectores de la población e implicaron una recomposición de lazos comunitarios.

En estos procesos, las mujeres fueron protagonistas centrales y contribuyeron a apuntalar la construcción colectiva de nuevas formas de vida, de comunidad, de barrios para la vida digna. Las movilizaba la vulneración de los derechos fundamentales para sostener la vida: alimentación, infraestructura de cuidado (jardines maternales, escuelas), vivienda y servicios básicos. Todas cuestiones que hacen a la reproducción de la vida. Así, la cuestión de las necesidades básicas para la vida, siempre resueltas dentro del ámbito doméstico, salían a la escena pública y política. De la casa a la ruta, a la movilización, a la ocupación de tierra, a la ocupación de una oficina estatal. Una vez más se hacía presente la pregunta de si esta irrupción pública del dolor privado podía transformar a las mujeres de las barriadas populares, a las piqueteras, en mujeres conscientes de su demanda de género. Eran mujeres que ocupaban un papel clave en la construcción de sus comunidades, partiendo desde un lugar de confrontación en muchos casos, y, en un sentido similar al de las Madres - que "salieron de sus cocinas para romper el silencio" - aquí lo hacían para ocupar el territorio público y las instituciones públicas, salir a la ruta y visibilizar sus demandas y, con ellas, la vulneración de sus derechos económicos y sociales. 
En este punto nos gustaría mostrar algunos fragmentos de alguna de las compañeras que han prestado testimonio para el proyecto de Insumisas y que han formado parte de estos procesos organizativos desde mediados de la década del $80 .^{1}$

Maisa Bascuas: El primer fragmento corresponde al testimonio de Cristina Coronel, una activista territorial de la localidad de Moreno (situada en el conurbano de la provincia de Buenos Aires), que ya, desde los años ochenta, se empezó a vincular con el proceso de construcción del movimiento de mujeres y feminista. Realizó un importante trabajo con las mujeres de los barrios populares, a la vez que se vinculaba con la APDH, en la incipiente Secretaría de la Mujer y sus Derechos. En esas dos intersecciones, ella apuntalaba el proceso de construcción popular en Moreno e, incluso, formaba parte de una toma en el barrio Latinoamericano de esa localidad.

El segundo fragmento que escuchamos es parte del testimonio de Lily Galeano. Ella es una referente en los procesos de ocupación de tierras desde mediados de los ochenta e hija de un militante sindical desaparecido durante la última dictadura. En 1986, Lily formó parte de la ocupación de tierras para la construcción del barrio “22 de enero” en La Matanza, proceso que tuvo mucha repercusión por aquellos años. Luego, participó en otros procesos que culminaron en la construcción de diferentes barrios en el conurbano bonaerense. A partir de estos fragmentos, podemos ver cómo las mujeres han sido protagonistas destacadas de estas acciones políticas: en muchos casos, liderando las tomas de tierra, asumiendo tareas de construcción y de organización del espacio y de la vida comunitaria. Y, al hacerlo, han contribuido a politizar las dimensiones de la reproducción de la vida. A la vez, ese mismo proceso las fortaleció y les permitió conectarse con un movimiento amplio de mujeres del cual comenzaron a participar de manera activa.

En este punto y como luego pudimos articular en otros testimonios para Insumisas, resulta interesante destacar la participación de estas mujeres en el Encuentro Nacional de Mujeres de 1986, el mismo año en que se producía la toma de tierras y la construcción del barrio "22 de enero" en La Matanza. Allí fueron Lily y sus compañeras del barrio; también, Cristina y sus compañeras del barrio Latinoamericano de Moreno. El $1^{\circ}$ Encuentro Nacional de Mujeres de 1986 se realizó en Capital Federal y fue un escenario relevante para la confluencia entre el movimiento de mujeres y el de derechos humanos La organización de ese encuentro ha tenido una historia propia que atravesó muchos meses de trabajo y conversaciones entre diferentes grupos. Las discusiones que se dieron alrededor del nombre del evento durante ese proceso organizativo marcaron el carácter de aquellas jornadas, pero además dejaron una impronta en el movimiento de mujeres y feministas, posible de rastrear hasta la actualidad.

La prioridad que han señalado quienes fueron parte de la comisión organizadora era la de encontrarse en un ámbito democrático y horizontal, donde todas las voces pudieran ser escuchadas, sin jerarquización de unas sobre otras. Esto las llevó a descartar un formato de congreso o conferencia para optar por un encuentro con talleres sobre temas específicos. Otra cuestión importante era poder construir una mirada de las problemáticas de las mujeres atenta a la diversidad territorial e inscripta en la realidad nacional. De allí el carácter federal del encuentro. Esta perspectiva se fortaleció con la propuesta de su rotación anual en distintos territorios. Los diferentes grupos que confluyeron en la organización compartían la caracterización de que había que trabajar por un encuentro que fuese masivo y que permitiera la construcción de una voz propia. De esta manera, el encuentro estaría abierto a todas las mujeres, sin vetos ni cupos por organización. De este conjunto de discusiones y criterios surgió el nombre: Encuentro Nacional de Mujeres. En aquellos años ochenta, el feminismo como identidad política estaba lejos de la masividad que adquiere en nuestros días.

En aquella comisión organizadora había mujeres que se identificaban como feministas, pero no todas se inscribían en esa tradición política. Sobre las feministas todavía predominaba una mirada estigmatizante, lo que explicaba la decisión de inscribir el encuentro en una perspectiva más amplia de las mujeres, en tensión pero articulada con la perspectiva feminista. Esta tensión productiva entre el movimiento feminista y el 
movimiento amplio de mujeres marcó el desarrollo de esa trama en Argentina. Si bien no todos los grupos feministas reconocían en ese momento la importancia de involucrarse en un proceso donde parte de la agenda feminista podía quedar restringida, la mayoría sí lo hicieron. Esto permitió que las mujeres de las barriadas populares, de las ocupaciones de tierra, de los "patios traseros de nuestras ciudades", se sintieran convocadas para llevar su voz, su experiencia colectiva pero singular.

En este punto, les queríamos mostrar un fragmento del testimonio de Cristina Coronel.2

Este fragmento nos muestra cómo el $1^{\circ}$ Encuentro Nacional de Mujeres convocó e intentó privilegiar el testimonio. Buscó construir una voz propia del movimiento, otorgándole a las mujeres provenientes de las luchas populares de los barrios periféricos un lugar muy importante. Por esa razón, estas mujeres se sintieron convocadas y encontraron en la construcción de este espacio, un lugar más para llevar su voz y para ampliar sus alianzas en un contexto muy particular.

En ese mismo encuentro, las Madres de Plaza de Mayo llevaron una serie de documentos con propuestas concretas para que se incluyeran en las conclusiones del ENM. Entre esas demandas, se encontraban: que el parlamento se pronunciara contra cualquier tipo de amnistía que implicara la impunidad a los responsables y ejecutores del secuestro y desaparición de miles de personas, de tortura, violación y el asesinato; que el parlamento declarara la desaparición de personas como crímenes de lesa humanidad; que se otorgara la libertad a Ilda Nava, presa política y a los demás presos políticos en Argentina. En ese documento, también destacaban la particularidad de la represión que sufrieron las mujeres:

No podemos seguir con las estadísticas; no se trata de fantasmas o números de legajo. Eran mujeres con inquietudes e ideales a
los que no se les dio oportunidad de defenderse en ningún juicio con abogados fiscal jueces. Mujeres que, como las Madres de
Plaza de Mayo después, habían contraído un compromiso con la vida en cada uno de sus frentes. Ese compromiso con la vida
sigue hoy más intenso y decidido que nunca. En los pañuelos blancos reverberan los nombres de tantas muchachas, de miles
de mujeres que de alguna forma buscan [...] una patria más solidaria y un destino más luminoso. (Documento de Madres de
Plaza de Mayo ante el Primer Encuentro Nacional de Mujeres (1986). Archivo Madres de Plaza de Mayo Línea Fundadora.)

Fueron varias las referentes de Madres de Plaza de Mayo y de Familiares que participaron de ese encuentro e incluso tuvieron un papel más significativo en su organización de la que el propio movimiento de derechos humanos reconoció en aquel entonces. Algunas importantes referentes fueron parte del Comité Organizador, otras fueron coordinadoras de talleres y relatoras en los distintos espacios de discusión y reflexión.

En esta búsqueda que promueve el proyecto Insumisas en Memoria Abierta por reconstruir y rescatar la memoria feminista y del movimiento de mujeres, hemos también recuperado una serie de materiales fundamentales para este archivo. Entre ellos, hay un afiche hermoso que nos acercó Lily y que lleva la consigna: "Si la vida no es digna, la dignidad es pelear para cambiarla" y en donde se ve una mujer con un niño en primer plano. También, gracias al archivo de Elsa Cola Arena, han aparecido un conjunto de casetes de audio, entre los cuales se encontraban grabadas las conclusiones de ese primer encuentro de 1986. Queríamos, para finalizar, compartir un fragmento de ese audio:

Que hemos venido a participar del taller Mujer y Familia en este encuentro. Integramos el grupo de 1200 familias ocupantes de un barrio que se llama " 22 de enero", en Matanza. Estamos aquí porque los medios de difusión nos marginan, la sociedad nos margina, porque nadie nos escucha. Por eso hemos venido a este encuentro, para hacerle saber a ustedes, mujeres, políticas, profesionales, amas de casa, a todas ustedes, qué es lo que nos pasa, por qué hemos tomado la tierra. Hemos tomado la tierra porque es la única alternativa que nos han dejado. Hemos tomado la tierra porque acá no se legisla para que el pueblo tenga vivienda. Porque hace muchos años que acá no se legisla para que el pueblo tengamos una vivienda digna, que es un derecho que nos da la constitución. Por eso hemos tomado la tierra, porque creemos que tenemos derecho a criar a nuestros hijos bajo un techo digno y propio, porque tenemos que escapar de desalojos cuando no tenemos para pagar un alquiler, porque venimos de zonas inundables, de villas miserias, porque estamos cansados de ser marginados, por eso hemos tomado la tierra... (Aplausos)

Acá se habló de mayor presupuesto para educación, para salud. Hay que hablar de un montón de cosas más. De nada nos sirve la educación si nuestros chicos no comen porque no pueden aprender entonces.

(Aplausos) 
Se habló de una deuda externa. Se habló que con esos intereses que pagamos por año se podrían construir 400 mil viviendas, que podríamos construir 12 mil escuelas, cuántas cosas podríamos hacer con lo que se paga con esa deuda externa. Y nos llaman usurpadores, ilegales ¿quién es más ilegal? ¿Quién le hace más daño al país? ¿Nosotros que tomamos tierra que nadie usaba ni para producir ni para hacer viviendas, o aquellos que nos dejaron en estas condiciones sin techo y que nos obligan a tomarla?

(Aplausos)

¿Quién es más culpable? Hay un dicho que dice que quien le roba a un ladrón tiene 100 años de perdón. No nos consideramos ladrones, estamos tomando lo que es nuestro, porque la tierra es de quien la produce y quien la ocupa (...) Esta intervención no estaba prevista en este encuentro, le pedimos disculpas a las organizadoras pero es la única forma que tenemos que nuestra voz y nuestra experiencia se conozcan. No vamos a dejar que se nos siga marginando, que se nos aísle. Que nadie se olvide que aquí, en los asentamientos, estamos haciendo parte de la historia argentina. Nada más y muchas gracias.

(Aplausos y ovación)

Lectura de las conclusiones del Taller y Familia del Primer Encuentro Nacional de Mujeres (1986). Fondo Elsa Cola Arena, Memoria Abierta.

\section{Muchas gracias}

Luego de las intervenciones, se abrió un interesante espacio para comentarios y consultas del público presente. Las preguntas y reflexiones compartidas pueden verse en el registro audiovisual del panel, a través de: Derecho UACh (9/9/2021). "Mesa 10 y Mesa 10B. II Conferencia Internacional: El lugar del archivo de las violencias de estado:". Youtube. https://www.youtube.com/watch?v=5gvwlncVXpc

\section{Notas}

1 Los testimonios de Cristina Coronel y Celia "Lily Galeano" se pueden ver el siguiente enlace: Derecho UACh (9/9/2021). “Mesa 10 y Mesa 10B. II Conferencia Internacional: El lugar del archivo de las violencias de estado:”. (Min. 57:20 a min. 59:20) Youtube. https://www.youtube.com/watch?v=5gvwlncVXpc

2 El testimonio de Cristina Coronel se pueden ver el siguiente enlace: Derecho UACh (9/9/2021). "Mesa 10 y Mesa 10B. II Conferencia Internacional: El lugar del archivo de las violencias de estado:”. (Desde minuto 1:06:05) Youtube. http s://www.youtube.com/watch?v=5gvwlncVXpc 